\title{
Estudo Clínico Epidemiológico das Fraturas da Coluna Vertebral
}

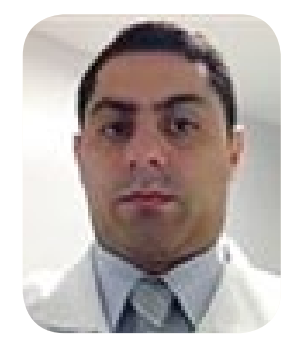

Rony Brito Fernandes ${ }^{1}$, Maurício Santos Gusmão², Djalma Castro de Amorim Júnior ${ }^{3}$, Maurício Guimarães Pimentel ${ }^{4}$, Tiago Argolo Bittencourt de Oliveira ${ }^{5}$, Marcos Almeida Mattos ${ }^{6}$

Artigo original: Fernandes RB; Gomes EG; Gusmão MS; Amorim DC; Simões MTV; Gomes
JF; Freire JB; Matos MA; Ajulay J; Pedreira AJS; Carvalho OEF. Clinical epidemiologica
spinal fractures. Coluna/Columna, vol.11 nº3. São Paulo July/Set 2012.

\section{INTRODUÇÃO}

As fraturas da coluna vertebral são importantes causas de morbidade e mortalidade nos dias atuais. É crescente o número de pacientes que chegam à emergência vítimas de lesões graves, podendo evoluir com sequelas irreversíveis, que atingem não só o paciente, mas também a família e a sociedade, levando a altos custos para o país. ${ }^{12}$

Com o aprimoramento do treinamento na conduta inicial da equipe de emergência e do atendimento pré-hospitalar, aumentou o número de pacientes com fraturas graves da coluna vertebral que não evoluem para o óbito imediato nos grandes centros brasileiros. A incidência de lesão neurológica no trauma raquimedular é de $40 \%$ em fraturas cervicais, $20 \%$ em fraturas torácicas e $15 \%$ nas fraturas lombares. ${ }^{1,2,3,7,9,10} \mathrm{E}$ mais frequente em pacientes do sexo masculino, por estes serem mais expostos a acidentes automobilísticos de alta energia, violência urbana como lesão por projétil de arma de fogo, queda de altura e mergulho em água rasa. ${ }^{1,2,}$

Nos EUA, 40 habitantes por milhão sofrem trauma da coluna vertebral, sendo que $15 \%$ a $20 \%$ desses pacientes evoluem com lesoes neurológicas, havendo um aumento gradual desse número a cada ano. ${ }^{1}$ Estes números, associados ao alto custo economico e social, transformam a lesão vertebral em um dos mais críticos problemas de saúde publica. Aliteratura cientifica existente no Brasil fornece pouca informação sobre dados epidemiológicos das fraturas vertebrais em seus diversos aspectos; este fato dificulta a implementação de políticas de prevenção e assistência aos pacientes acometidos por este trauma.

O objetivo desse estudo foi avaliar o perfil clínico-epidemiológico do trauma da coluna vertebral nos pacientes atendidos na emergência do Hospital Geral do Estado, em Salvador-Bahia, e identificar potenciais fatores associados ao prognóstico destas lesões.

\section{MATERIAL E MÉTODOS}

Realizado o estudo de revisão de prontuário, retrospectivo, analisando dados clínicos e epidemiológicos dos pacientes atendidos no Hospital Geral do Estado da Bahia (HGE), no periodo de 1991 a 2010. As características avaliadas foram idade, sexo, tipo de lesão, mecanismo de trauma, procedência, nivel vertebral lesado, tratamento e escala de Frankel para avaliação de déficit neurológico. O HGE é o centro de referência de trauma raquimedular de todo o Estado da Bahia, recebendo pacientes regulados de outras cidades baiahas e também casos de emergência que procuram diretamente a unidade de TRM deste hospital.

Todos os pacientes com suspeita de fratura da coluna vertebral foram criteriosamente examinados $\mathrm{e}$ realizados inicialmente radiografias simples. Em caso de fraturas, foram submetidos ao exame de tomografla computadorizada com reconstrução tridimensional para melhor avaliação das lesões ósseas. Se possível, neste momento, estas lesōes foram classificadas e emitida conduta se tratamento cirúrgico ou conservador. Em casos de dúvida quanto à estabilidade ou necessidade de avaliação das compressões medulares foi realizado exame de ressonancia magnetica.

Nos prontuários foram pesquisados dados a respeito de idade, sexo, procedencla, mecanismo de trauma, tipo da lesão, nivel da lesão, escala de Frankel da admissão e pos-cirurgico, tratamento e complicações. Estes dados são padronizados na unidade como essenciais para 0 atendimento dos pacientes, sendo obrigatorio o registro por parte do médico que atende o TRM. Por este motivo, foi possivel recuperar todos os dados de 1.917 pacientes com fratura da coluna vertebral submetidos a tratamento no HGE no período de 1991 a 2010. Os dados foram colhidos também nos relatórios de alta, que continham variáveis citadas acima. Os dados foram tabulados por meio de estatística descritiva, utilizando proporções para variáveis discretas e médias com desvio-padrão para variáveis contínuas. Todas as variáveis foram analisadas separadamente e as associações ou correlações importantes foram cruzadas e examinadas em busca de significância estatística. As informações contidas no banco de dados foram armazenadas e analisadas nos softwares Excel 2003 e Stastistical Package for the Socia Sciences-SPSS, versão 11.0. O teste do qui-quadrado foi utilizado para comparação entre variáveis discretas e o teste $t$ de Student foi utilizado no caso de variáveis continuas. Foram considerados estalisticamente ficativos aqueles resultados com o $p<0,05$.

\section{RESULTADOS}

Este estudo somou 1.917 pacientes, sendo 606 com fratura em mais de um nível na coluna cervical, 237 com fratura em mais de um nível torácico e 79 com fratura em mais de um nível lombar. A maioria do sexo masculino, $85,2 \%$ ( $n=1.633)$, com uma relação de 5,75:1. A média de idade foi de 34 anos, variando desde 3 anos a mínima e 92 anos a máxima, com $3,8 \%$ perdidos. A faixa etária mais acometida foi adultos com 20 a 29 anos $(30,2 \%)$, seguida da faixa entre 30 e 39 anos (25,9\%), e observou-se a redução desta prevalência com aumento da idade. (Figura 1)

A procedência desses pacientes foi da zona urbana em $69,2 \%$ ( $n=1.327$ ) dos casos; zona rural, $25,4 \%$ $(n=486) ;$ e sem informação no prontuário foi de $5,4 \%$ $(n=104)$.

Os mecanismos de trauma mais frequentes foram queda de altura, 40,4\% ( $n=774)$; acidente automobilístico, 22,9\% ( $(n=439)$, fratura por projetil arma de fogo (PAF), 8,2\% ( $n=158)$; mergulho em água rasa, $8 \%$ $(n=154)$; acidente motociclistico, $6,7 \%(n=129) ;$ queda da própria altura, $2,8 \%(n=53)$; outros, $6,7 \%(n=128) ; e$ $4,3 \%(n=82)$ sem informação no prontuário.

O tipo mais frequente são fraturas isoladas, $75,6 \%(n=1.449)$; seguido por mielopatia cervical, $5,5 \%(n=106)$, luxação sem associação a fratura ossea, $4,3 \%(n=83)$; sciwora, 0,3\% (n=7); outros, 5,3\% $(\mathrm{n}=102)$, e sem informaçáa no prontuario, $9 \%(\mathrm{n}=172)$. 0 tratamento cirurgico foi indicado em $88,2 \%$ $(n=1.691)$ dos casos, o conservador, em 7,2\% ( $n=138)$ e sem informação, 4,6\% ( $n=88$ ).

Em $61,7 \%(n=1182)$ não houve complicações pós-cirúrgicas, sendo que $12,2 \% \quad(n=234)$ apresentaram úlcera por pressão na região sacral, $6,4 \%(n=122)$ com infecção do trato urinário (ITU), 1,6\% (n=30) com trombose venosa profunda (TVP), 1,3\% ( $n=25)$, infecções respiratorias, $1,2 \%(n=23)$, infecçoes da ferida operatória, $0,6 \%(n=12)$ foram a óbito, $0,3 \%(n=5)$, fraturas de outros ossos associadas, $1,1 \%(n=22)$ e sem informações no prontuário, 3,7\% ( $n=262)$. (Tabela 1) Em relação ao nível vertebral mais acometido, a vertebra $L 1$ fol acometida em 11,4\% $(n=219)$ dos casos, seguida de $\mathrm{C} 5,4,5 \%(\mathrm{n}=86) ; \mathrm{T} 12,4 \%(\mathrm{n}=76) ; \mathrm{C} 2$, $3 \%(n=58) ; L 2,2,2 \%(n=42)$ e demais listadas na tabela 2. Mais de uma vertebra fraturada na regiăo cervical ocorreu em $31,6 \%(n=606)$ dos casos. Na região torácica, $12,4 \%(n=237)$ e na região lombar, $4,1 \%(n=79)$. Nas tranş̧⿸厂 e T12-L1 5,9\% ( $n=114)$ dos casos

Os pacientes foram examinados quanto ao déficit neurologico na sua admissão, usando a escala de Frankel e os resultados obtidos foram descritos conorme figura 2.

Na relação entre mecanismo de trauma, escala de Frankel e sexo se evidenciou que o sexo masculino foi mais frequente em todos os mecanismos de trauma na seguinte proporção: em queda de altura, $80,1 \%$ $(\mathrm{n}=522)$; em acidente automobilístico, 83,7\% ( $\mathrm{n}=314)$; em PAF, $97 \%(n=32)$; e nos casos de mergulho em água rasa, $98,5 \%(n=131)$, sendo estes valores estatisticamente significantes (Tabela 3).

Em relação à escala de Frankel e ao mecanismo de trauma, a maioria dos pacientes vítimas de queda de altura, $75,6 \%(n=151)$, foram classificados na admissão como Frankel $\mathrm{E}$, acidente automobilístico, $75 \%$ $(n=180)$, como Frankel E, PAF, 77,4\% ( $n=24)$, também como Frankel $E$, enquanto em mergulho em água rasa, $34,3 \%(n=36)$, foram Frankel B, com valores estatisticamente significantes (Tabela 3).

As relações entre mecanismo de trauma e a média da idade foram: queda de altura teve uma média de idade de 37,3 anos ( $n=636)$; acidente automobilístico, $33,6$ anos ( $n=357) ;$ PAF, 27,2 anos $(n=30)$; e mergulho em água rasa, 24,4 anos ( $n=133)$, encontrando valores significativos $(p<0,001)$ (Tabela 4$)$.

\section{DISCUSSÃO}

Este estudo avaliou 1.917 pacientes que foram atendidos na emergencia do $\mathrm{HGE}$, centro de referencia de TRM no estado da Bahia, e nenhum outro estudo publicado teve avaliação desta quantidade de pacientes. Foram avaliados pacientes atendidos entre 1991 e 2010, 20 anos, e são poucos os serviços de trauma raquimedular no Brasil que têm tal casuística. Em relação ao sexo acometido, a grande maioria são homens, cerca de $85 \%$ dos casos compativeis com as publicaçōes cientificas internacionais e nacionais. A maioria da procedência desses pacientes foi da zona urbana, não havendo publicação com esta variável. 
Em relacão ao mecanismo de trauma, o mais frequente foi a queda de altura, seguido por acidente automobilístico não apresentando mudanças em relação à maioria das publicações.

Puertas \& Gomes, em 1998, constataram que a queda de altura, seguida de mergulho em água rasa, foi o mecanismo de trauma mais trequente das lesões traumáticas da coluna cervical. ${ }^{15}$

Gonçalves et al. evidenciaram que o mecanismo de trauma mais prevalente foi de queda de altura, principalmente queda de laje, seguido por acidente automobilístico. ${ }^{15}$ Montesano e Berson destacaram que a causa mais frequente das lesoes da coluna vertebral tambem săo acidentes automobilisticos, seguidos por queda de altura, esportes e outros, mas ressaltam que a queda de altura representa $70 \%$ dos casos em pacientes acima de 75 anos. ${ }^{1}$

Neste estudo, a relação do mecanismo de trauma com a média da idade evidenciou que TRM causado por mergulho em água rasa atinge uma população mais jovem, com idade media de 24 anos, e queda de altura com media de 37 anos. O fato e que pacientes mais jovens fazem mais atividades recreativas, como mergulho em rios e lagos de baixa profundidade, principalmente em cidades com clima mais tropical, como é SalvadorBahia. A queda da própria altura, mais comum em idosos, encontra-se na variável queda de altura.

Neste estudo se evidenciou que a maioria das lesões foram fraturas isoladas, que podiam estar associadas ou não a mais de um nivel. Houve poucos casos de mielopatia, Sciwora e luxaçoes isoladas. Em seu estudo, Sekon et al. mostraram uma incidência maior de fraturas desviadas $(40 \%)$, seguida de fraturas do tipo burst $(30 \%)$ e poucos casos de Sicwora $(5 \%)$." A grande maioria das fraturas, após exame e avaliação dos criterios de instabilidade, $88 \%$ dos casos foram abordados cirurgicamente. Como neste estudo foram computados pacientes desde 1991, foram usadas técnicas de fixação vertebral mais antiga, como Hartshill ate o moderno arsenal terapeutico com parafusos pediculares. Em seu estudo, Koch et al. relataram um predominio de tratamento cirurgico na maioria dos casos, $51 \%$ quando queda de altura e $32 \%$ quando acidente automobilístico. ${ }^{3}$ No estudo de Zaninelli et al. $60,9 \%$ da sua casuística, o tratamento feito foi conservador com colete de Jewett ou imobilização gessada antigravitacional, repouso, analgésicos e acompanhamento ambulatorial. ${ }^{1,3}$

Em $61,7 \%$ dos casos não houve complicações pós-cirúrgicas, sendo que as principais complicações foram úlcera por pressão na região sacral e infecção do trato urinário, dados estes importantes para o cirurgião coluna vertebra.

Onivel vertebral mais acometido foi a vértebra de L1 em $11,4 \%$, seguido da vértebra cervical C5 e torácica T12, confimando pela lleratura cientifica nacional e internacional. Hu et al. relataram em seus estudos que $L 1$ fol a vertebra mais acometida, seguida de $L 2$ e T12. Zaninelli et all relataram que $50 \%$ das fraturas localizavam na região T12 a L2.' Esse nivel de T12 e L1 e mais suscetivel, pelo fato de ser uma area de transiçáo rigida para uma flexivel. Santos et all descreveram que $40 \%$ das fraturas ocorrem na região de -C6. ${ }^{14,15}$

Os pacientes foram examinados, de acordo a escala de Frankel na sua admissão, com os resultados no gráfico 1. Em $45 \%$ dos casos, os pacientes foram classificados como Frankel $E$, seguidos de Frankel $B$. Fo et al. tambem evidenciaram que Frankel $E$ foi o mais comum, seguido de Frankel $D$ A, sendo o Frankel B o menos acometido.

Em relação à escala de Frankel e ao mecanismo de trauma, este estudo evidenciou um predomínio do Frankel $B$ nos pacientes com causa de mergulho em agua rasa, sendo este o déficit neurológico mais comum nas lesões da região cervical."

\section{CONCLUSÃO}

Pacientes jovens, com média de idade de 34 anos, do sexo masculino, procedentes da zona urbana $e v$ timas de queda de altura ou acidente automobilistico, e a população de risco para fratura e lesões medulares na coluna vertebral. Quando a causa foi merguIho em água rasa, está mais associado as lesões na região cervical e tende a apresentar déficit neurológco. O nivel vertebral mais acometido foram as vertebras de T12 a L2 na transição toracolombar e C5-C6 na cervical. A vertebra de $L 1$ foi mais acometida sem déficit neurológico (Frankel E) na maioria dos casos. Na maioria dos casos se fez necessário o tratamento cirúrgico, com poucas complicaçōes pós-operatorias.

Os autores sugerem mudanças nas politicas púb cas, principalmente na area urbana, com campanh educacionais e de prevenção para quedas de altura cautela no mergulho em água rasa e de acidentes auomobilísticos e motociclísticos.

\section{TABELAS}

Tabela 1 - Distribuição das características dos traumas de coluna vertebral atendidos no serviço de emergência do Hospita Geral do Estado, dura o peíodo de 1901 a 2010, em Salvador - Bahia $(n=1917)$

\begin{tabular}{|c|c|c|c|c|c|}
\hline Variáveis & $\mathrm{N}$ & $\%$ & Variáveis & $\mathrm{N}$ & $\%$ \\
\hline \multicolumn{3}{|l|}{ Procedência } & \multicolumn{3}{|l|}{ Sexo } \\
\hline Rural & 486 & 25,4 & Feminino & 284 & 14,8 \\
\hline rbana & 1327 & 69,2 & Masculino & 1633 & 85,2 \\
\hline Sem informação & 104 & 5,4 & $\begin{array}{l}\text { Mecanismo de } \\
\text { Trauma }\end{array}$ & & \\
\hline \multicolumn{3}{|l|}{ Faixa Etária } & $\begin{array}{l}\text { Acidente } \\
\text { automobilístico }\end{array}$ & 439 & 22,9 \\
\hline $0-9$ anos & 9 & 0,5 & $\begin{array}{l}\text { Acidente } \\
\text { motocicilistico }\end{array}$ & 129 & 6,7 \\
\hline $10-19$ anos & 238 & 12,4 & PAF & 158 & 8,2 \\
\hline $20-29$ anos & 530 & 27,6 & $\begin{array}{l}\text { Mergulho água } \\
\text { rasa }\end{array}$ & 154 & 8,0 \\
\hline 30 - 39 anos & 448 & 23,4 & Queda de altura & 774 & 40,4 \\
\hline $40-49$ anos & 318 & 16,6 & $\begin{array}{l}\text { Queda da } \\
\text { propria altura }\end{array}$ & 53 & 2,8 \\
\hline 50 - 59 anos & 176 & 9,2 & Outros & 128 & 6,7 \\
\hline 60 anos ou mais & 126 & 6,6 & Sem informação & 82 & 4,3 \\
\hline Sem informação & 72 & 3,8 & Tipo de trauma & & \\
\hline \multicolumn{3}{|l|}{ Tratamento } & Sciwora & 5 & 0,3 \\
\hline Conservador & 138 & 7,2 & $\begin{array}{l}\text { Mielopatia } \\
\text { cervical }\end{array}$ & 106 & 5,5 \\
\hline Cirúrgico & 1691 & 88,2 & atura & 1449 & 75,6 \\
\hline Sem informação & 88 & 4,6 & Luxação & 83 & 4,3 \\
\hline \multicolumn{3}{|l|}{ Complicą̧ōes } & Outros & 102 & 5,3 \\
\hline Ausente & 1182 & 61,7 & Sem informação & 172 & 9,0 \\
\hline ITU & 122 & 6,4 & Niveis & & \\
\hline $\begin{array}{l}\text { Infecção } \\
\text { respiratória }\end{array}$ & 25 & 1,3 & Cervical (C1-C7) & 242 & 12,5 \\
\hline $\begin{array}{l}\text { Úlcera por } \\
\text { pressão }\end{array}$ & 234 & 12,2 & $\begin{array}{l}\text { Mais de um nivel } \\
\text { cervical }\end{array}$ & 606 & 31,6 \\
\hline TVP & 30 & 1,6 & C7-T1 & 34 & 1,8 \\
\hline Infecção & 23 & 1,2 & $\begin{array}{l}\text { Torácica (T1- } \\
\text { T12) }\end{array}$ & 184 & 9,8 \\
\hline $\begin{array}{l}\text { Fraturas } \\
\text { associadas }\end{array}$ & 5 & 0,3 & $\begin{array}{l}\text { Mais de um nivel } \\
\text { torácico }\end{array}$ & 237 & 12,4 \\
\hline Óbito & 12 & 0,6 & 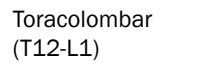 & 114 & 5,9 \\
\hline Outro & 22 & 1,1 & Lombar (L1-L5) & 304 & 15,8 \\
\hline \multirow[t]{2}{*}{ Sem informação } & 262 & 3,7 & $\begin{array}{l}\text { Mais de um nível } \\
\text { lombar }\end{array}$ & 79 & 4,1 \\
\hline & & & Sem Informação & 117 & 6,1 \\
\hline
\end{tabular}

Tabela 2 - Distribuicão absoluta e percentual dos niveis actes da coluna vertebral dos pacientes atendidos no servico de emergência do Hospital Geral do Esde 1991 a 2010 em Salvador - Bahia ( $n=1917)$

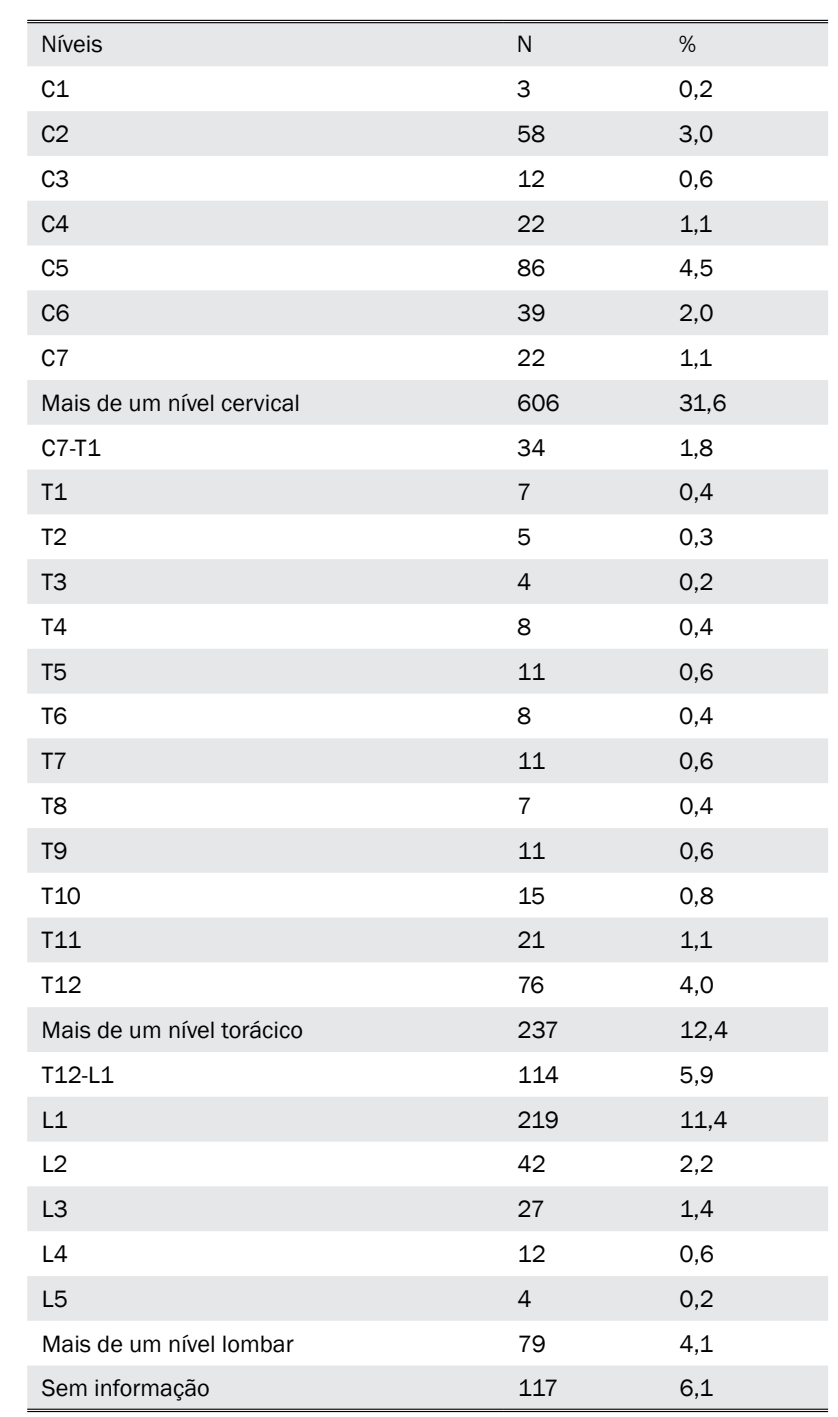


Tabela 3 - Distribuição do mecanismo de trauma mais frequente dos pacientes com fraturas da coluna vertebral, atendidos no serviço de emergência do Hospital Geral do Estado, durante o período de 1991 a 2010, segundo as variáveis sexo e Frankel, Salvador - Bahia

\begin{tabular}{|c|c|c|c|c|c|c|c|c|c|}
\hline \multicolumn{10}{|c|}{ Mecanismo de Trauma } \\
\hline \multirow{2}{*}{ Variáveis } & \multicolumn{2}{|c|}{ Queda de altura } & \multicolumn{2}{|c|}{$\begin{array}{l}\text { Acidente } \\
\text { automobilístico }\end{array}$} & \multirow{2}{*}{$\mathrm{N}$} & \multirow{2}{*}{$\begin{array}{l}\text { PAF } \\
\%\end{array}$} & \multicolumn{2}{|c|}{ Mergulho água rasa } & \multirow[b]{2}{*}{ Pvalor } \\
\hline & $\mathrm{N}$ & $\%$ & $\mathrm{~N}$ & $\%$ & & & $\mathrm{~N}$ & $\%$ & \\
\hline \multicolumn{10}{|c|}{ Sexo $(n=1406)$} \\
\hline Feminino & 130 & 19,9 & 61 & 16,3 & 1 & 3,0 & 2 & 1,5 & \multirow{2}{*}{$<0,001$} \\
\hline Masculino & 522 & 80,1 & 314 & 83,7 & 32 & 97,0 & 131 & 98,5 & \\
\hline \multicolumn{10}{|c|}{ Frankel na Admissão (n=979) } \\
\hline A & 15 & 3,5 & 12 & 5,0 & 2 & 6,5 & 12 & 11,4 & \multirow{5}{*}{$<0,001$} \\
\hline B & 51 & 11,8 & 22 & 9,2 & 2 & 6,5 & 36 & 34,3 & \\
\hline$c$ & 16 & 3.7 & 16 & 6,7 & 1 & 3,2 & 20 & 19,0 & \\
\hline D & 24 & 5,5 & 10 & 4,2 & 2 & 6,5 & 8 & 7,6 & \\
\hline $\mathrm{E}$ & 328 & 75,6 & 180 & 75,0 & 24 & 77,4 & 29 & 27,6 & \\
\hline
\end{tabular}

P-valor $<0,05$ pelo Teste do Qui-Quadrado de Pearson

Tabela 4 - Distribuição do mecanismo de trauma mais frequente dos pacientes com fraturas da coluna vertebral, atendidos no serviço de emergência do Hospital Geral do Estado, durante o período de 1991 a 2010, segundo média de idade, Salvador - Bahia

\begin{tabular}{|c|c|c|c|c|c|c|c|c|c|c|c|c|c|}
\hline \multicolumn{14}{|c|}{ Mecanismo de Trauma } \\
\hline \multirow[b]{2}{*}{ Variável } & \multicolumn{3}{|c|}{ Queda de altura } & \multicolumn{4}{|c|}{ Acidente automobilístico } & \multicolumn{3}{|l|}{ PAF } & \multicolumn{3}{|c|}{ Mergulho água rasa } \\
\hline & N & média & $\begin{array}{l}\text { Desvio } \\
\text { Padrao }\end{array}$ & $\mathrm{N}$ & média & $\begin{array}{l}\text { Desvio } \\
\text { Padrăo }\end{array}$ & $\mathrm{N}$ & média & $\begin{array}{l}\text { Desvio } \\
\text { Padrăo }\end{array}$ & N & média & $\begin{array}{l}\text { Desvio } \\
\text { Padrao }\end{array}$ & P Valor \\
\hline Idade & 636 & 37,3 & 13,6 & 357 & 33,6 & 12,0 & 30 & 27,2 & 10,0 & 133 & 24,4 & 9,2 & 0001 \\
\hline
\end{tabular}

$\begin{array}{cccc}\text { ldade } & 636 & 37,3 & 13,6 \\ { }^{*} P \text {-valor }<0,05 & \text { pelo Teste de ANOVA }\end{array}$

\section{FIGURAS}

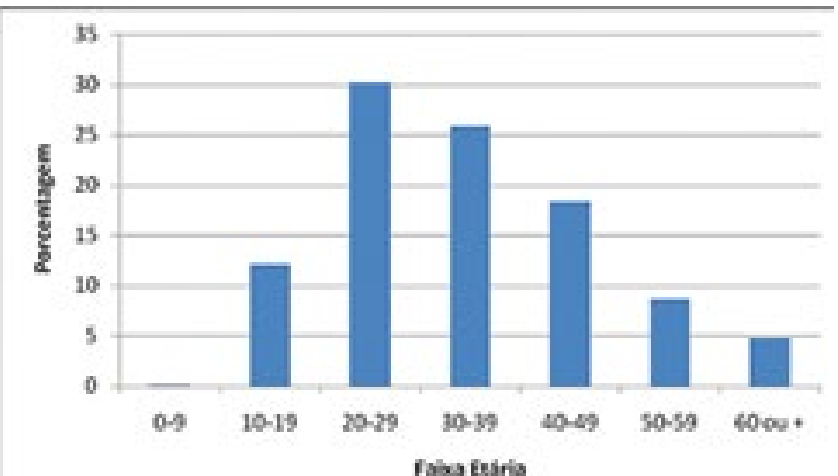

Figura 1 - Distribuição das fraturas de coluna vertebral atendidas no serviço de emergência do Hospital Geral

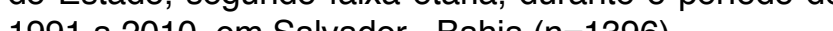

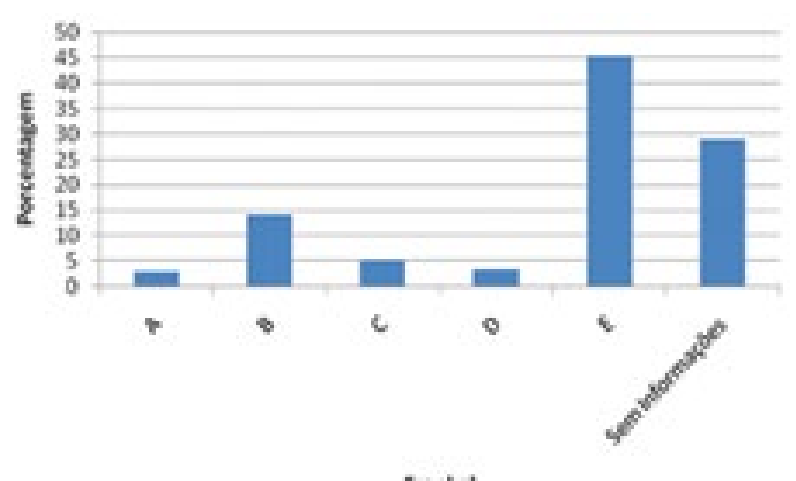

Figura 2 - Distibuição dos traumas de coluna vertebral conforme o déficit neurológico no momento de atendiSalvador - Bahia $(n=1917)$. mento inicial, no serviço de emergência do Hospital Ge-

\section{REFERÊNCIAS}

1. Zanielli, E. M. et al.; Avaliação Epidemiológica das Fraturas Toracica e Lombar em pacientes atendidos no Pronto Socorro do Hosplical do Trabalhador da URR de Curtiba-Paraná. Rev. Coluna/Columna 2005; 4:11-15. 2. Philip J. B. et al.; Incidence, Epidemiology, and res among U.S. Army Aviators. Journal Trauma 2001; 50:855-861

3. Koch, A.; Zanielli, E. M.; Graellis, X. S.; Epidemiologia de fraturas da coluna de acordo com o mecanismo de trauma: análise de 502 casos. Rev. Coluna/ Columna 2007; 6:18-23.

4. Herbert S, Xavier R, Pardini Jr ArlindoG, Barros Filho TEP. Ortopedia e traumatologia: princípios e prática. $3^{\mathrm{a}}$ ed. Porto Alegre. Artmed, 2003.

5. Bucholz, R. W.; Heckman, J. D.; Rockwood e Green Fraturas em Adultos, RW editors. $5^{a}$ ed. São Paulo: Manole; 2006 .p. 1295-97.

6. Vaccaro, A. R.; Betz, R. R.; Zeidman, S. M.; Cirurgia da Coluna- Princípios e Prática, $1^{\mathrm{a}}$ ed. Rio de Janeiro:Dilivros; 2007. p. 407-83.

7. Defino, H. L. A.; Lesões Traumáticas da Coluna Vertebral, $1^{a}$ ed. São Paulo:Bevilacqua; 2005. p. 53-153. 8.Pereira, A. F. F. et al.; Avaliação epidemiológica das fraturas da coluna torácica e lombar dos pacientes atendidos no Serviço de Ortopedia e Traumatologia do Hospital Getúlio Vargas em Recife-PE. Rev. Coluna/ Columna 2009;8(4):395-400.

9. Anderle, D. V. et al.; Avaliação epidemiológica dos pacientes com traumatismo raquimedular operados no Hospital Estadual Professor Carlos da Silva Lacaz. Rev. Coluna/Columna 2010,9(1):58-61.

10. Rodrigues, L. C. L.; Bortoletto, A; Matsumoto, M. H.; Epidemiologia das fraturas torocalombares cirúrgicas na zona leste de São Paulo. Rev. Coluna/ Columna 2010;9(2):132-137.

1- Serviço de Ortopedia do Hospital Santa Izabe

Endereço para correspondência:

ronybritofernandes@hotmail.com 